\title{
Knowledge Disclosure as Intellectual Property Rights Protection*
}

\author{
Carlos J. Ponce ${ }^{\dagger}$
}

\begin{abstract}
We study a model in which an inventor discloses knowledge about its innovation and then a rival chooses the probability of attaining a competing invention. Disclosures, by creating prior art, diminish the probability that the rival has of receiving a patent for its invention (legal externality), but, by revealing knowledge, they decrease the marginal cost of R\&D (knowledge externality). We stress the following result. If the knowledge externality is large compared to the legal externality, decreasing the patentability standards leads to fewer disclosures and may hinder R\&D. We also determine the impact of changes in market payoffs on the equilibrium level of disclosures and R\&D.
\end{abstract}

Keywords: Disclosure, patentability standard, independent invention, transfer effect, threat effect.

\footnotetext{
* I am grateful to Joseph Ostroy, David Levine and Bryan Ellickson for their support and insights. I also want to thank Leeat Yariv, Chrysovalantou Milliou, seminar participants at UCLA, University Carlos III de Madrid and especially Marco Celentani for many helpful comments and suggestions. All errors are my own.

${ }^{\dagger}$ Department of Economics, Universidad Carlos III de Madrid, Calle Madrid 126, 28903 Getafe (Madrid), SPAIN; cjponce@eco.uc3m.es
} 


\section{Introduction}

The knowledge that first inventors disclose in patents is usually difficult to protect. Future inventors may use it to obtain superior non-infringing innovations that may end up undermining the market position of the original innovator. This is likely the crucial reason for an important majority of real life patents being obscure and difficult to read despite the formal enablement requirement of patent law (see, Scotchmer [16]). Similarly, inventors, to avoid disclosure and imitation may use secrecy. In this manner, they protect their superior market position. ${ }^{1}$ However, the 'secret' may be independently rediscovered by a second research firm. Furthermore, at least in the U.S. if the research firm that rediscovers the invention obtained a valid patent, it would have the right to exclude the prior inventor from using the secret innovation. ${ }^{2}$

In these two 'disclosure problems', innovators anticipate that disclosure reveals knowledge that future rivals may potentially use to obtain alternative better products that later might be protected by patents. Thus, they may be reluctant to disclose because as a result they could be hurt by stronger rivals in downstream competition. But choosing secrecy undermines one of the main goals of the patent system: the disclosure of innovative knowledge. However, on the other hand, we point out that first inventors also foresee that rivals invest in $R \& D$ to obtain their own inventions. Thus, the competitive threat of future inventions drives innovators to disclose their knowledge to diminish the probability that a rival may obtain a patent for a related invention. The idea behind this observation is simple: because future patents are evaluated in the light of the prior art, first inventors, by disclosing, and creating new prior art make it more difficult for second inventors to obtain future patent rights on related innovations. ${ }^{3,4}$

In this paper, we explore the strategic determination of disclosure when inventors are confronted with this trade-off. We examine the optimal $R \& D$ response of a rival to disclosure by an inventor and the impact of changes in patent policy and market payoffs on the equilibrium configuration of disclosure and R\&D intensity. Our ideas are organized around the primitive that if the rival is successful in his $\mathrm{R} \& \mathrm{D}$ and then receives a patent for its invention, the innovator obtains a lower profit than otherwise.

Disclosure plays a dual role: (a) disclosure reveals valuable knowledge that the rival uses in its R\&D activity; and (b) disclosure creates prior art and thus it diminishes the probability that the rival

\footnotetext{
${ }^{1}$ Secrecy has gained prominence is several US industries (Cohen, Nelson and Walsh [7], and Lerner [12]) specially among smaller firms. It is a fact that for these type of firms the costs of detecting misappropriation and enforcing patent rights are significant (Lanjouw and Schankerman's [11] and Lerner [12]).

${ }^{2}$ See Denicolo and Franzoni [7] for a discussion of the first inventor defense.

${ }^{3}$ Prior art is all the public knowledge either in previous patents, manuscripts, printed publications, etc. that existed prior to the filing of a patent application. In the United States, when an innovator discloses her invention, a one-year grace period ensures that the innovator's patent right is not immediately extinguished.

${ }^{4}$ An illustrative example of disclosures in the public domain is that of Plantronics, a telephone headset manufacturer in Santa Cruz, California. The company developed a new technology for reducing microphone noise. However, the invention couldn't be used right away. Plantronics posted a "description" of it on the web site to establish the legal existence of the idea. Another case is that of The Nutter Machine Company, in Ohio, that has published at least part of one of its inventions on the web site. See "Protecting Intellectual Property" The New York Times 02/18/2002, "Suddenly, 'Idea Wars' Take On a New Global Urgency" The New York Times 11/11/2002, "On the Defensive About Invention" The Financial Times 09/19/2001. It follows from the example that secrecy is not inconsistent with disclosures or publishing. Innovators may find attractive to choose a mixture of secrecy and publishing.
} 
has of receiving a patent right. Concerning this last point, notice that, according to patent law, an invention can be protected by a patent only if it is a substantial advancement over the prior state of the art. In reality, however, matters are more complicated and sometimes patents are granted and then subsequently invalidated in private court disputes. The most frequent ground for invalidity is prior art and, in many cases, a printed publication accessible to the public is enough to invalidate a patent (see Allison and Lemley [1]). For the sake of simplicity, we do not make any distinction between the process of obtaining a patent and the defense from challenges to their validity subsequently made by competitors.

Within this setup, we address questions like the following ones: (i) Is decreasing the patentability standards a good policy to encourage disclosure and to boost future R\&D activities? (ii) How do disclosure and $\mathrm{R} \& \mathrm{D}$ intensity depend on the market premium for technological leadership?

Understanding our findings requires to consider the following two observations. First, we capture the idea that disclosure reveals valuable knowledge by assuming that it decreases the rival's marginal cost of achieving any probability of success in its R\&D activity. This knowledge externality encourages R\&D. But, by creating prior art, disclosure decreases the probability that the rival has of receiving a patent. This legal externality, on the contrary, discourages R\&D. In this paper, we deal with the non-trivial case in which the knowledge externality prevails over the legal externality. The resulting effect is that the rival's best response $R \& D$ probability increases with disclosure. We call this net positive impact of disclosures on R\&D the transfer effect. In this case the marginal cost of disclosing is positive and a higher knowledge externality leads to a higher transfer effect and a higher marginal cost of disclosing.

Second, the marginal benefit of disclosure increases in the level of the R\&D probability of the rival. We call this impact of the $R \& D$ probability on disclosure the threat effect. Intuitively, disclosure are only used to prevent the rival from patenting a competing idea. But to receive a patent, the rival must first succeed in its R\&D activity. When the level of the R\&D probability is higher, success and hence the threat of the rival's patent is a more likely event. Thus, the marginal value of disclosing rises.

In answering our first question we find that a decrease in the standards of patentability is not always an appropriate policy to promote knowledge dissemination and to foster R\&D. Observe the channels through which a lower patentability standard affects the equilibrium. First, when the patentability standard decreases the marginal cost of disclosing increases because the rival's patenting probability is higher for any given disclosure level. However, a lower patentability standard also raises the marginal because the rival's best response R\&D probability shifts upwards and thus, the threat of the rival's patent becomes a more likely event. This causes an increase in the marginal benefit of disclosure.

When the knowledge externality is small compared to the legal externality (in a sense to be made precise later) the threat effect prevails over the transfer effect and a decrease in the patentability standards generates a higher level of disclosure and boosts R\&D. This is the only case in a lower patentability standard, overall, has a positive effect on R\&D.

However, on the other side of the coin, a decrease in the patentability standards results in a lower level of disclosures when the knowledge externality is at least twice as large as the legal external effect of 
disclosures. Or equivalently, it generates a strategic withholding of knowledge when the transfer effect is large. But then the issue of whether or not this policy encourages R\&D does not have a clear-cut answer. For a fixed disclosure level, $R \& D$ intensity raises as a result of a decrease in the patentability standard. But a lower patentability standard induces fewer disclosures, increasing the rival's marginal cost of $\mathrm{R} \& \mathrm{D}$ and thus discouraging $\mathrm{R} \& \mathrm{D}$. The final outcome is that when the knowledge externality is large relative to the legal externality, that is when the social value of disclosure is high, this policy may hinder R\&D. Of course, the strategic withholding of knowledge will damage other economic sectors and firms which might find useful the know how related to the invention.

This result contributes to the debate about patentability standards and patent quality. It suggests in some knowledge-based industries most likely in Software, Semiconductors and biotechnology there are good reasons to believe that, disclosure, innovation and $\mathrm{R} \& \mathrm{D}$ overall would be well promoted by increasing the patentability standards. Why? Because in these sectors knowledge disclosure seems to be crucial for decreasing the future cost of incremental innovations (high knowledge externality) while the impact of disclosure on the patentability of future patents is really low. This has been documented in the 'low' quality of patents that most of the time do not meet the requirement of patentability, particularly with respect to prior art (which is dispersed in the public domain rather than in prior patents).

Second and finally, we determine the effects of an exogenous increase in the difference in profits that a firm obtains when it is the only one which uses the most advanced technology (or innovation) in the market compared to the case in which both firms use it, i.e., an increase in the market premium for technological leadership (or simply, market premium). This is an interesting exercise that attempts to find a regularity between the amount of secrecy, the intensity of R\&D and the size of the premium for leadership. We show that for those industries in which the knowledge externality is relatively small or moderate compared to the legal externality, a higher market premium leads to a higher level of disclosure and R\&D intensity. This result contradicts the common view that those industrial sectors with a high market premium should be characterized by a low level of disclosure and 'big' secrets. In this case a 'flourishing' environment characterized by 'high-stakes-high-disclosure and high R\&D activity' arises. But we also underscore that when the knowledge externality is high enough the picture might look exactly the opposite: 'high-stakes-low-disclosure and low R\&D activity'. In this case, 'big' secrets may substantially hurt future innovation.

The 'disclosure problem' has been studied from very different perspectives. Our paper owes much to Denicolo and Franzoni [7]. We share with them the view that prior innovators may be hurt by a subsequent inventor. Their focus, however, is on welfare analysis and they do not consider, as we do, the possibility of prior art creation. Scotchmer and Green [15] is also related to our work. They focus on the impact of patent policy on the incentives to both innovate and disclose intermediate discoveries. The main difference between the papers is that in our model the motivation to disclose is rooted in the possibility of blocking a rival's patent, an aspect not examined in their paper. This paper is also related to a literature which explores defensive publications in patent races. Baker, Lichtman and Mezzetti [4] and Bar [5] construct models in which firms disclose in order to prolong the race, and this 
gives followers a chance to catch up. These papers consider patent races and disclosure is executed by laggards rather than by leaders (first inventors) as in the current paper. ${ }^{5}$ Anton and Yao [2 and 3] and Johnson [9] have also explored the disclosure problem. Anton and Yao [2 and 3] examined information disclosure to signal strong capability in an environment of limited IP and asymmetric information . The analysis of Johnson [9] is close to ours because both papers deal with the same broad issue: the blocking, by an innovator, of a potential rival's patent. However, the main goal of Johnson's paper is to identify under which circumstances a defensive publication strategy is preferred by an innovator to the IP choices of secrecy and patenting. On the contrary, our goal is to examine the problem of disclosure by an innovator and the R\&D incentives of her rival. Finally, although, we do not examine the choice of IP by the original innovator, it may be easily incorporated along the lines of Ponce [14] and our results would remain exactly the same.

The paper proceeds as follows. Section II sets forth the basics of our model and provides some preliminary results. Section III presents our main findings. In Section IV some limitations and extensions to our basic setup are discussed. Section V concludes. All the proofs are provided in the Appendix.

\section{The Model and Preliminary Results}

Our model contains the following elements. There are two firms, an innovator, referred to as 'she' and denoted by $A$, and a rival, referred to as 'he' and denoted by $B$. We assume that $A$ has obtained an innovation that may be a new technology that lowers the unit cost of production or an improvement in the quality of a given commodity. The extensive form of the game is as follows.

(i) $A$ chooses a disclosure level, $d$, from her disclosure set, $\mathcal{D}:=[0,1]$. Disclosures are technological knowledge regarding the innovation. They may be executed in a patent or in the public domain, for example, in a publication. When $d=0, A$ chooses to keep her innovation entirely hidden and when $d=1, A$ makes the best possible description of the innovation. Partial disclosures, $d \in(0,1)$, are also possible.

(ii) After observing $d, B$ chooses a probability of obtaining a related innovation (or simply, the R\&D probability) $f \in[0,1]$. We observe that if $B$ is successful in his $\mathrm{R} \& \mathrm{D}$, he will try to obtain a patent to use his innovation. We assume that $B$ 's innovation does not infringe on the previous patent, if $A$ has chosen to obtain one. Of course, if disclosures are in the public domain this observation is irrelevant. Thus, the only resolution about property rights that must be made is whether $B$ is granted a patent or not.

(iii) If $B$ succeeds in his R\&D, the Patent Office decides whether or not to grant a patent to $B$. After that decision every residual uncertainty about property rights vanishes. If $B$ receives a patent, he will exclude $A$ from using the new technology in the market competition game. ${ }^{6}$

\footnotetext{
${ }^{5}$ Parchomovsky [13] was the first to draw attention to the possibility of strategically creating prior art. Litchman, Baker and Kraus [12], offered a signalling model of defensive publication.

${ }^{6}$ We do not make any distinction between the process of awarding patents and the challenges to their validity frequently made by competitors. However, the assumption that the decision about property rights is concluded before market competition fits, for instance, a patent reexamination request. This type of procedure deals mainly with issues of patentability related to prior patents and printed publications.
} 
(iv) $A$ and $B$ engage in duopolistic competition. The payoffs the innovator and his rival obtain in the market competition game depend on the R\&D and patenting outcomes. Each possible event is represented by a pair $(R D, P)$, with the first element standing for the result of the $\mathrm{R} \& \mathrm{D}$ and the second one for the property right resolution. For example, the pair $(S, F)$ stands for the event in which $\mathrm{R} \& \mathrm{D}$ is a success for the rival, but he fails to obtain a patent.

For simplicity we posit a symmetric payoff structure. If the rival fails in his R\&D activity then the innovator will have the monopoly over the superior technology. In this contingency, profits will be $\pi^{H}$ for the innovator and $\pi^{L}$ for her rival. On the other side of the coin, if the rival succeeds in obtaining a superior technology and gets a patent over it, profits will be $\pi^{H}$ for the rival and $\pi^{L}$ for the innovator. Finally, if the rival succeeds in obtaining a superior technology but he fails in getting a patent, both firms will obtain $\pi^{M}$. We assume that payoffs satisfy the following ordering: $\pi^{H}>\pi^{M}>\pi^{L}$. This assumption is satisfied in many typical models of industrial organization: Cournot with homogeneous commodities and Bertrand with differentiated product to name just two.

Equilibrium. It is important to notice that although $A$ discloses private knowledge about an innovation, our game is one of perfect information. Formally, every information set of every player is a singleton. Thus, the equilibrium concept for our game is subgame perfect Nash equilibrium (SPE). We concentrate on pure strategy SPE. A strategy for player $A$ is a choice of disclosure, $d$, from her disclosure set $\mathcal{D}$ and a strategy for $B$ is a real-valued function $f:[0,1] \longrightarrow[0,1]$.

\section{A. RED by the Rival}

Let $C(f, d)$ denote $B$ 's cost of achieving a probability $f$, when the disclosure level is $d$. We assume that $C(f, d)$ satisfies the usual conditions: $\forall(f, d) \in[0,1] \times[0,1]: C(f, d) \geq 0, C_{f}(f, d) \geq 0, C_{f f}(f, d)>$ 0. Also: $\forall d \in \mathcal{D}: C(0, d)=0, C_{f}(0, d)=0$. More important:

ASSUMPTION 1: $\forall(f, d) \in[0,1] \times[0,1]: C_{f d}(f, d)<0$.

Assumption 1 says that a higher disclosure level diminishes the marginal cost of duplication. It implies that $C_{d}(f, d)<0$. The role of this assumption will be clear when we state Lemma 1 .

Before continue, it is useful to specify the chance for $B$ of obtaining a patent. This is captured by Assumption 2. As a preliminary, let $\gamma(d ; \bar{s})$ for any $d \in \mathcal{D}$ denote the probability that $B$ receives a patent when $A$ 's disclosure level is $d$ and let $\bar{s}$ be a measure of the patentability standards. The main assumption about $\gamma(.,$.$) is: { }^{7}$

ASSUMPTION 2: (a) $\forall d \in \mathcal{D}: \gamma(d) \in(0,1)$.

(b) $\forall d \in \mathcal{D}: \gamma_{d}(d)<0$ and $\gamma_{d d}(d)>0$.

(c) $\forall d \in \mathcal{D}: \frac{\partial \gamma}{\partial \bar{s}}>0$

The crucial parts are (b) and (c). On the one hand, part (b) holds that disclosure has a marginal decreasing effect on the probability of securing a valid patent. On the other hand, part (c) says, given $B$ 's innovation, a bigger $\bar{s}$ increases $B$ 's probability of receiving a patent, for any given disclosure level

\footnotetext{
${ }^{7}$ In general, derivatives will be denoted by subscripts.
} 
(i.e., for any level of prior art). Thus, an increase in $\bar{s}$ can be seen as a decrease in the patentability standards.

Now we can solve $B$ 's problem. For a given $d, B$ chooses $f$ to maximize his expected payoff. With probability $1-f \mathrm{R} \& \mathrm{D}$ is a failure and $B$ 's profits are $\pi^{L}$. With probability, $f, \mathrm{R} \& \mathrm{D}$ is a success. In this case, with probability $\gamma(d ; \bar{s}), B$ obtains a patent and gets $\pi^{H}$ and with probability $1-\gamma(d ; \bar{s})$, a patent is not granted and he obtains $\pi^{M}$. Given that $B$ pays $C(f, d)$ whether he succeeds or not, $B$ 's expected payoff is: $E U^{B}\left(f, d, \pi^{B}, \bar{s}\right)=\pi^{L}+f\left\{\gamma(d ; \bar{s})\left(\pi^{H}-\pi^{M}\right)+\left(\pi^{M}-\pi^{L}\right)\right\}-C(f, d)$. To avoid a corner solution at $f=1$, we suppose that at $d=1: C_{f}(1, d)>\left[\gamma(d ; \bar{s})\left(\pi^{H}-\pi^{M}\right)+\left(\pi^{M}-\pi^{L}\right)\right] .8$ Thus, $B$ 's best response is determined by the following necessary and sufficient first order condition:

$$
\frac{\partial E U^{B}(f, d, \pi, \bar{s})}{\partial f}=\gamma(d ; \bar{s})\left(\pi^{H}-\pi^{M}\right)+\left(\pi^{M}-\pi^{L}\right)-C_{f}(f, d)=0
$$

Observe that, conditional on success in $\operatorname{RED} D, \Delta_{V P}^{B}:=\left(\pi^{H}-\pi^{M}\right)$ is the difference in profits that $B$ attains by being successful in obtaining a patent. Thus, we call $\Delta_{V P}^{B}$ the value of the patent. Also, conditional on a failure in obtaining a patent, $\Delta_{V I}^{B}:=\pi^{M}-\pi^{L}$ is the difference in profits that $B$ obtains by being successful in his $\mathrm{R} \& \mathrm{D}$. Hence, we call $\Delta_{V I}^{B}$ the value of the innovation. In Lemma 1 we prove the existence of $B$ 's best response and characterizes its basic properties.

LEMMA 1: (a) B's best response exists and it is a $C^{1}$ function $f(d, \bar{s}, \pi)$

(b) $B$ 's best response function $f(d, \bar{s}, \pi)$ is such that:

$$
\frac{\partial f(d, \bar{s}, \pi)}{\partial d}=\left(C_{f f}\right)^{-1}\left[\gamma_{d}(d ; \bar{s}) \Delta_{V P}^{B}-C_{f d}(f, d)\right] \lessgtr 0
$$

and:

$$
\frac{\partial f(d, \bar{s}, \pi)}{\partial \Delta_{V P}^{B}}>0, \frac{\partial f(d, \bar{s}, \pi)}{\partial \Delta_{V I}^{B}}>0, \frac{\partial f(d, \bar{s}, \pi)}{\partial \bar{s}}>0
$$

PROOF. Part (a) follows from the satisfaction of the conditions for the implicit function theorem: $\forall(f, d) \in[0,1] \times[0,1]: C_{f f}(f, d) \neq 0$. Part (b) follows from the characterization of comparative static effects of $d, \Delta_{V P}^{B}, \Delta_{V I}^{B}$ and $\bar{s}$ on $f^{*}$ using the first order condition. The specific formulas can be found in the Appendix.

Part b) of Lemma 1 has two different implications. First, it shows that when either $\Delta_{V P}^{B}$ or $\Delta_{V I}^{B}$ is higher, $B$ will choose a higher $f$. Similarly, if patentability standards are decreased, a higher $f$ will be chosen, for a given disclosure level.

Second, it shows that an increase in $d$ can lead to either a higher or a lower level of $f(d, \bar{s}, \pi)$. This follows from the combination of two opposing external effects: the knowledge externality and the legal externality. The first externality is formally captured by $B$ 's lower marginal cost of obtaining any given $f$ for a higher disclosure level: $C_{f d}(f, d)$. The second external effect corresponds to $B$ 's lower

\footnotetext{
${ }^{8}$ It follows from the structure of this stage that it is never a best response for $B$ to choose a zero R\&D probability.
} 
marginal expected return because his probability of patenting is smaller when disclosure is higher and it is analytically defined as: $\gamma_{d}(d ; \bar{s}) \Delta_{V P}^{B}$.

If the legal externality is large relative to the knowledge externality, higher disclosure will decrease $B$ 's best response. Given that, conditional on success, the probability of patenting also decreases with disclosure, it follows that the equilibrium disclosure strategy is $d^{*}=1$. For the rest of the paper, we focus on the case in which the knowledge externality dominates the legal externality and the net effect of disclosure is to enhance $B$ 's R\&D capability. Thus, from now on we suppose that $\frac{\partial f\left(d, \bar{s}, \pi^{B}\right)}{\partial d}>0 .{ }^{9}$ We call this net positive impact of disclosure on $\mathrm{R} \& \mathrm{D}$ the transfer effect.

\section{B. Disclosure by the Innovator}

$A$ chooses a disclosure level $d \in \mathcal{D}$, anticipating $B$ 's best response function. Thus, we can write $A$ 's problem as:

$$
\begin{aligned}
& \max _{(f, d) \in K} E U^{A}(f, d)= \max _{(f, d) \in K}\left[(1-f) \pi^{H}+f W\left(d, \pi^{A}, \bar{s}\right)\right] \\
& \text { where: } W(d, \pi, \bar{s}):=\gamma(d ; \bar{s}) \pi^{L}+(1-\gamma(d ; \bar{s})) \pi^{M} \\
& K \quad: \quad=\left\{(f, d) \in[0,1] \times[0,1]: \gamma(d ; \bar{s}) \Delta_{V P}^{B}+\Delta_{V I}^{B}=C_{f}(f, d)\right\}
\end{aligned}
$$

$W(d, \pi, \bar{s})$ is $A$ 's expected payoff in the state in which $B$ is successful in his R\&D. $A$ 's problem reflects her trade-off when choosing a level of $d$. By disclosing, the probability of being at the technological edge, $1-f$, is reduced. But if $B$ were successful in his R\&D, $A$ 's expected payoff would be higher because $\frac{\partial W(d, \pi, \bar{s})}{\partial d}=-\gamma_{d}(d ; \bar{s})\left(\pi^{M}-\pi^{L}\right)>0$.

\section{Equilibria: Existence and Uniqueness}

Given Lemma 1, it is sufficient to focus on $A$ 's problem to show our elementary existence and uniqueness result.

Proposition 1: (a) A SPE exists.

(b) If (i) $B$ 's best response, $f(d, \bar{s}, \pi)$, is a strictly convex function of disclosure; and (ii). A's payoff function, $E U^{A}(f, d)$ is quasiconcave, then: a unique SPE exists.

PROOF. See the Appendix

For the rest of the paper, we deal with the case of a unique interior equilibrium. We denote the equilibrium disclosure strategy by $d^{*}$ and by $f^{*}:=f\left(d^{*}, \pi, \bar{s}\right)$ the equilibrium R\&D probability. Let $M B$ and $M C$ denote the marginal benefit and marginal cost of disclosing respectively. In an interior equilibrium $d^{*}$ and $f^{*}$ are such that: ${ }^{10}$

$$
M B^{*}:=-\gamma_{d}\left(d^{*} ; \bar{s}\right) f\left(d^{*}, \pi, \bar{s}\right) \Delta_{P R}^{A}=\frac{\partial f}{\partial d}\left(d^{*}, \pi, \bar{s}\right)\left[\Delta_{M P}^{A}+\gamma\left(d^{*} ; \bar{s}\right) \Delta_{P R}^{A}\right]:=M C^{*}
$$

\footnotetext{
${ }^{9}$ Formally, we assume that

$\forall(f, d) \in[0,1] \times[0,1]:-C_{f d}(f, d)>-\gamma_{d}(d ; \bar{s}) \Delta_{V P}^{B}$

${ }^{10}$ We derive the marginal benefit and cost of disclosing in the Appendix.
} 
where $\Delta_{P R}^{A}:=\pi^{M}-\pi^{L}$ and $\Delta_{M P}^{A}:=\pi^{H}-\pi^{M}$. Equation (3) underscores the main motivations that lead to disclosing and the links between disclosures and R\&D intensity. Thus, it is worthy to discuss it a little bit.

First, notice that, conditional on success in RED (or simply, the successful state), the term $\pi^{M}-\pi^{L}$ represents the extra profits that $A$ obtains when $B$ fails to obtain a patent compared to the case in which he succeeds in obtaining one. Thus, $\pi^{M}-\pi^{L}$ captures the additional profits $A$ obtains from successfully blocking $B$ 's patenting activities. Thus, we call $\Delta_{P R}^{A}:=\pi^{M}-\pi^{L}$ the preemption return.

With that interpretation in hand, observe that the marginal benefit of disclosing corresponds to the increase in A's expected payoff in the successful state due to higher disclosures times the R\&D probability. This observation simply points out that the marginal benefit of disclosure increases in the level of the RED probability and therefore it implies that any exogenous perturbation that increases player $B$ 's strategy, $f(d, \pi, \bar{s})$, will also result in an increase of $A$ 's disclosure strategy. This is what we call the threat effect.

Second, conditional on a failure in B's patenting activity, the term $\pi^{H}-\pi^{M}$ represents the extra profits the innovator obtains when the rival fails in his R\&D compared to the case in which he succeeds. Thus, $\pi^{H}-\pi^{M}$ represents the extra profits $A$ makes due to holding the technological leadership. We call $\Delta_{M P}^{A}:=\pi^{H}-\pi^{M}$ the market premium for technological leadership (or simply, market premium). Also, using our definition of $\Delta_{P R}^{A}$, A's expected payoff in the successful state can be expressed as: $W(d, \pi, \bar{s})=\pi^{M}-\gamma(d ; \bar{s}) \Delta_{P R}^{A}$. Similarly, when $B$ fails in his R\&D, $A$ 's payoff is: $\pi^{H}$. Thus, we define $A$ 's lost payoff due to $B$ 's success in $\mathrm{R} \& \mathrm{D}$ as: $\Pi(d, \bar{s}, \pi):=\pi^{H}-W(d, \pi, \bar{s})=\Delta_{M P}^{A}+\gamma(d ; \bar{s}) \Delta_{P R}^{A}$. Hence, using $B$ 's best response function we have that $f(d, \pi, \bar{s})\left[\Delta_{M P}^{A}+\gamma(d ; \bar{s}) \Delta_{P R}^{A}\right]$ is $A$ 's expected lost payoff due to $B$ 's R\&D.

Using these intuitions, notice that the marginal cost of disclosing corresponds to the increase in A's expected lost payoff due to the higher R\&D probability associated with a higher disclosure level.

\section{Main Results}

\section{A. A Policy Experiment: A decrease in the Patentability Standards}

The view of the Patent Office and courts towards patents and disclosure is determined in our model by the commonly known parameter $\bar{s}$. Our patent decision rule determines that $\frac{\partial \gamma(d ; \bar{s})}{\partial \bar{s}}>0$. In words, given $B$ 's invention, a higher $\bar{s}$ increases the probability that $B$ has of receiving a patent for a given disclosure level (i.e., for a given level of prior art). Thus, an increase in $\bar{s}$ can be seen as a decrease in the patentability standards. The following proposition characterizes the equilibrium response of both disclosures and R\&D intensity to an increase in $\bar{s}$.

PROpOsition 2: (a) If and only if $-C_{f d}\left(f^{*}, d^{*}\right)>-2 \gamma_{d}\left(d^{*}, \bar{s}\right) \Delta_{V P}^{B}$ a higher $\bar{s}$ causes the equilibrium level of disclosures $d^{*}$ to decrease. The equilibrium level of the R\&D probability $f\left(d^{*}, \pi, \bar{s}\right)$ may either decrease or increase.

(b) If $-C_{f d}\left(f^{*}, d^{*}\right) \leq-2 \gamma_{d}\left(d^{*}, \bar{s}\right) \Delta_{V P}^{B}$ a rise in $\bar{s}$ causes the equilibrium R\&D probability $f\left(d^{*}, \pi, \bar{s}\right)$ to increase. 
PROOF. See the Appendix

Changes in the patentability standards affect disclosure through two different channels. First, an increase in $\bar{s}$ raises the marginal value of disclosures. The reason for this is as follows. A higher $\bar{s}$ increases $B$ 's marginal returns of choosing a higher $\mathrm{R} \& \mathrm{D}$ probability. In other words, $B$ 's best response shifts upward. Thus, by the threat effect, an increase in $\bar{s}$ raises the marginal benefit of disclosing. But, a decrease in the patentability standards also increases the marginal cost of disclosing by increasing, for any given disclosure level, $B$ 's patenting probability. The magnitude of the increment in the marginal cost of disclosing is proportional to the size of transfer effect.

The proposition solves the above trade-offs and it generates interesting policy implications. In particular, it shows that when the knowledge externality is small compared to the legal externality (i.e., when the inequality of part b) holds) a decrease in the patentability standards generates a higher level of disclosures and boosts R\&D. This is the only situation in which an argument in favor of implementing this type of policy can be sustained.

However a decrease in the patentability standards results in a lower level of disclosures when the knowledge externality is at least twice as large as the legal external effect of disclosures. Or equivalently, this type of policy generates a strategic withholding of knowledge on the part of the innovator when the transfer effect is large. But then the issue of whether or not an increase in $\bar{s}$ is a good policy to encourage $\mathrm{R} \& \mathrm{D}$ does not have a clear-cut answer. Keeping the disclosure level fixed, the $\mathrm{R} \& \mathrm{D}$ probability shifts upward as a result of an increase in $\bar{s}$. But a higher $\bar{s}$ leads to lower disclosure, increasing $B$ 's marginal cost of $\mathrm{R} \& \mathrm{D}$ and thus decreasing the incentives to $\mathrm{R} \& \mathrm{D}$. The final result is that when the knowledge externality is large relative to the legal externality, that is when the social value of disclosure is high because of its effects in diminishing the marginal cost of achieving further innovations, a higher $\bar{s}$ may hinder R\&D.

This result shows that, in most cases, the design of an optimal patent policy should include 'strict' patentability standards. In this case initial innovators, who are usually the owners of key knowledge for future incremental innovations, would find in their own interest to disclose valuable social knowledge that shall constitute the technological base for future innovations. This policy 'advice' is even more appropriate for industries that are knowledge-based like Computers, Software and Semiconductors, for example.

\section{B. Comparative Statics}

Here we study the consequences of exogenous changes on disclosures and R\&D. We are interested in examining the response of disclosures and $\mathrm{R} \& \mathrm{D}$ to changes in the market premium and in the preemption return. In particular we seek to answer the following two questions:

(a) How does an increase in the market premium for technological leadership affect disclosures and $\mathrm{R} \& \mathrm{D}$ ?

(b) How does an increase in the preemption return affect disclosures and $\mathrm{R} \& \mathrm{D}$ ? 
The answer to the first question is provided in Proposition 3. Our main interest in answering question (a) lies in understanding the regularities between the incentives to keep the technological leadership by withholding knowledge by the innovator, and the incentives to invest in $\mathrm{R} \& \mathrm{D}$ by the rival to achieve that leadership.

Question (b) is answered in Proposition 4. As we said before, the preemption return is the additional profits $A$ obtains from successfully blocking $B$ 's patenting activities. Our main concern here is related to the situation in which higher duopolistic profits, or equivalently less intense product market competition when both players use the same innovation, leads to more or less disclosures and R\&D.

\section{B1. Changes in the Market Premium}

Notice that the market premium and the value of the patent to $B$ are the same, that is $\Delta_{M P}^{A}=$ $\pi^{H}-\pi^{M}=\Delta_{V P}^{B}$. The proposition below studies the impact of a higher $\Delta_{M P}^{A}$ on disclosures and R\&D.

PROpOSITION 3: (a) If and only if $-C_{f d}\left(f^{*}, d^{*}\right)>-2 \gamma_{d}\left(d^{*}, \bar{s}\right) \Pi\left(d^{*}, \bar{s}, \pi\right)$ an increase in $\Delta_{M P}^{A}$ causes the equilibrium level of disclosures $d^{*}$ to decrease. The equilibrium level of the R\&D probability $f\left(d^{*}, \pi, \bar{s}\right)$ may either decrease or increase.

(b) If $-C_{f d}\left(f^{*}, d^{*}\right) \leq-2 \gamma_{d}\left(d^{*}, \bar{s}\right) \Pi^{A}\left(d, \bar{s}, \pi^{A}\right)$ an increase in $\Delta_{M P}^{A}$ causes the equilibrium level of the R\&D probability $f\left(d^{*}, \pi, \bar{s}\right)$ to increase.

PROOF. See the Appendix

The understanding of this result follows from the next two observations. On the other hand, let us suppose that the best response probability of the rival $f\left(d^{*}, \pi, \bar{s}\right)$ is kept fixed. Then changes in $\Delta_{M P}^{A}$ do not affect the marginal benefit of disclosing. The reason is simple: if $f\left(d^{*}, \pi, \bar{s}\right)$ does not change, the threat effect would be absent and the marginal benefit of disclosing does not change. Therefore, keeping fixed $f\left(d^{*}, \pi, \bar{s}\right)$, an increase in $\Delta_{M P}^{A}$ raises only the marginal cost of disclosing because it increases $A$ 's lost profits when $B$ succeeds in his R\&D. This increment in the marginal cost of disclosing is proportional to the size of the transfer effect. Therefore, $d^{*}$ diminishes. Given that $B$ 's best response is kept fixed and disclosure diminishes, the equilibrium $R \& D$ probability would also decrease.

The intuition until now should be clear: if $B$ 's best response did not change, $A$ would disclose less to increase her probability of being the technological leader and thus to capture the higher profits associated to that market position.

On the other, however, an increase in $\Delta_{M P}^{A}$ corresponds to an increment in $\Delta_{V P}^{B}$ and this raises the marginal benefit of disclosing. The argument is simple. When $\Delta_{V P}^{B}$ increases, $B$ does optimally responds by shifting upward his $\mathrm{R} \& \mathrm{D}$ probability for any given disclosure level. Then, by the threat effect, a higher best response R\&D probability raises the marginal benefits of disclosing. ${ }^{11}$ With

\footnotetext{
${ }^{11}$ There is a second reinforcing effect that increases disclosure. An increase in $\Delta_{V P}^{B}$ decreases the marginal cost of disclosing by diminishing the extent of the transfer effect (i.e., by leading to a less steep response of the R\&D probability to disclosures). The reason for this second effect is simple. A higher $\Delta_{V P}^{B}$ increases the absolute value of the legal externality that $A$ imposes on $B$ and thus the optimal change in the R\&D probability to disclosures diminishes. However it is of a second order of importance.
} 
respect to $\mathrm{R} \& \mathrm{D}$ intensity, a rise in $\Delta_{V P}^{B}$ shifts $B$ 's best response upward and this increment in the $\mathrm{R} \& \mathrm{D}$ probability is strategically reinforced by larger disclosure on the part of $A$.

Thus overall what determines whether disclosures increases or not are the relative magnitudes of the threat effect and the transfer effect. Part b) of the proposition conveys the intuitive idea that when the knowledge externality is not so large, the threat effect dominates the latter and hence an increase in $\Delta_{M P}^{A}$ leads to a higher level of disclosure. The higher level of disclosure plus the exogenously larger $\Delta_{V P}^{B}$ determine a higher equilibrium R\&D probability.

This result challenges the common view that when the market premium for leadership is higher $A$ should disclose less in order to increase her probability of keeping the technological edge. The main idea behind it is that when the market premium increases $B$ invests so intensively in $\mathrm{R} \& \mathrm{D}$ and hence the marginal value of disclosing raises a lot! If $B$ 's best response reacts intensely to changes in the market premium (i.e., to changes in $\Delta_{V P}^{B}$ ) then the equilibrium level of disclosure increases.

But, we also observe that if the knowledge externality is sufficiently large (i.e., if the condition of part a) holds) a higher market premium results in strategic withholding of knowledge on the part of the innovator and this increases the marginal cost of $R \& D$. The final outcome may be that a higher market premium causes a lower level of R\&D intensity, a result that also seems counterintuitive.

\section{B2. Changes in the Preemption Return}

Notice that the preemption return and the value of the innovation for the rival are the same, that is: $\Delta_{P R}^{A}=\pi^{M}-\pi^{L}=\Delta_{V I}^{B}$. Thus, it is direct to confirm that the following proposition holds.

PROPOSITION 4: An increase in $\Delta_{P R}^{A}$ causes the equilibrium level of disclosures $d^{*}$ and the $\mathrm{R} \& \mathrm{D}$ probability $f\left(d^{*}, \pi, \bar{s}\right)$ to rise.

PROOF. See the Appendix

Proposition 4 can easily be understood. On the one hand, an increase in $\Delta_{P R}^{A}$ increases the extra profits $A$ obtains by creating new prior art. This increases the marginal benefit of disclosing and therefore encourage disclosure. Also, because an increase in $\Delta_{P R}^{A}$ coincides with an increase in $\Delta_{V I}^{B}$, it results that the increment in $\Delta_{V I}^{B}$ raises $B$ 's marginal return of doing $\mathrm{R} \& \mathrm{D}$ and thus his best response shifts upward. Hence, by the threat effect, a higher $\Delta_{V I}^{B}$ leads also to a higher marginal benefit of disclosing. These two forces reinforce each other to increase disclosure and the equilibrium $R \& D$ probability.

On the other hand, an increase in $\Delta_{P R}^{A}$ increases $A$ 's lost profits when $B$ succeeds in $\mathrm{R} \& \mathrm{D}$ and thus raises the marginal cost of disclosing. The increment in the marginal cost of disclosing, as shown by equation (3), is proportional to the size of the transfer effect.

The proposition shows that, at $d^{*}$, the marginal benefit of disclosing increases more than the marginal cost of disclosing, and thus the equilibrium disclosure strategy increases. The equilibrium R\&D probability must also increase. This results confirms that if the duopolistic competition is not so intense when both players use the same technology disclosure and R\&D are higher. 


\section{Main Results: Summary}

The table below summarizes our main results. We classify them according to the size of the knowledge externality. We identify three different regimes. The Low regime corresponds to the case in which: $-\gamma_{d}\left(d^{*}, \bar{s}\right) \Delta_{V P}^{B}<-C_{f d}\left(f^{*}, d^{*}\right)<-2 \gamma_{d}\left(d^{*}, \bar{s}\right) \Delta_{V P}^{B}$. In this case, it can be seen from the table that a decrease in the patentability standards leads to a higher disclosure and $R \& D$. This is the only case that clearly supports an argument in favor of implementing this type of policy. A higher market premium is also associated to a higher level of disclosure and $\mathrm{R} \& \mathrm{D}$. The Intermediate regime holds when: $-2 \gamma_{d}\left(d^{*}, \bar{s}\right) \Delta_{V P}^{B} \leq-C_{f d}\left(f^{*}, d^{*}\right) \leq-2 \gamma_{d}\left(d^{*}, \bar{s}\right) \Pi(d, \bar{s}, \pi)$. In this regime a rise in $\bar{s}$ decreases disclosure and might have exactly the opposite intended effect of fostering R\&D; while a higher market premium induces more knowledge disclosure and encourages R\&D intensity. Finally, the High regime holds when: $-C_{f d}\left(f^{*}, d^{*}\right)>-2 \gamma_{d}\left(d^{*}, \bar{s}\right) \Pi(d, \bar{s}, \pi)$. For this case, an increase in $\bar{s}$ has the same qualitative impact on disclosures and $\mathrm{R} \& \mathrm{D}$ that in the Intermediate regime. However, an increase in $\Delta_{M P}^{A}$ may finally lead to lower levels of R\&D.

$\begin{array}{cccc}\uparrow \bar{s} \Rightarrow & \text { Low regime } & \text { Intermediate regime } & \text { High regime } \\ \uparrow \Delta_{M P}^{A}=\uparrow \Delta_{V P}^{B} \Rightarrow & d^{*} \uparrow, f^{*} \uparrow & d^{*} \downarrow, f^{*} ? & d^{*} \downarrow, f^{*} ? \\ \uparrow \Delta_{P R}^{A}=\uparrow \Delta_{V I}^{B} \Rightarrow & d^{*} \uparrow, f^{*} \uparrow & d^{*} \uparrow, f^{*} \uparrow & d^{*} \downarrow, f^{*} ? \\ & & d^{*} \uparrow, f^{*} \uparrow & d^{*} \uparrow, f^{*} \uparrow\end{array}$

\section{Discussion}

Our model is based on a number of simplifying assumptions that prove to be useful to understand the interactions between disclosures, R\&D, patent policy and market payoffs' perturbations. However, some details of the underlying innovation-disclosure game between the innovator and her rival and some other features of the patenting environment deserve additional discussion. This section fills that void.

1. Asymmetric Information: Our innovation-disclosure game is one of complete information. However, asymmetric information may be introduced into the model by assuming that the innovator might have private information about the type of innovation that she obtained. Along these lines, suppose that there are two types of innovations represented by their 'size' (i.e. for example the magnitude of the cost reduction from the old technology): $\Delta \in\{\underset{-}{\Delta}, \bar{\Delta}\}$ where $0<\Delta<\bar{\Delta}$ and assume that a bigger size implies higher profits. The game would become a signaling game and disclosure would play two different roles (a) creating prior art and (b) signaling to the rival the profits that he most likely would obtain in downstream competition.

One reasonable conjecture is that if the rival's random variable $\Delta$, conditional on having being successful in R\&D, is positively correlated with the size $\Delta$ for the innovator, and the threat effect is not too high, bigger (i.e., more profitable) innovations should be less disclosed. The intuition just comes from the idea that disclosure would help the rival to increase investment in $R \& D$ when the rival realizes that the probability of discovering a profitable innovation is high; something that is detrimental to the innovator's profit. However this intuition relies on the assumption that the threat effect is not 
too high; because if it were sufficiently large then the marginal value of disclosure would be so high that a potentially signaling equilibrium with the innovator disclosing more the bigger the size of the innovation may exist.

2. Subsequent Innovation by the Original Innovator: We have assumed that our innovator does not attempt to discover the second innovation. This assumption, which is in line with the literature of sequential innovation, is usually justified by assuming that the rival was the only one who has a private idea to develop the second innovation. Though extending the model to include a race between the innovator and the rival would complicate the analysis, our conjecture is that it will not change the main conclusions.

The model might be extended in the following direction. Both the innovator and the rival would choose the probability of obtaining the second innovation, $f_{i} \in[0,1] \forall i \in\{A, B\}$. Now the $\mathrm{R} \& \mathrm{D}$ cost would be: $C(f, d ; \theta)$; where $\theta \in \Theta$ represents the 'quality' of the idea to develop the second innovation. Thus if $\theta^{\prime}>\theta \Longrightarrow C\left(f, d ; \theta^{\prime}\right)<C(f, d ; \theta)$ and $\exists \theta \in \Theta: \forall(f, d), C(f, d ; \theta)=\infty$. Thus our original model would be a particular case in which for the innovator $C(f, d ; \theta)=\infty$. Then a necessary condition for disclosures to exist is that $\theta^{B}-\theta^{A}>0$ and a simple argument can be used to show that $\exists \theta^{*}$ such that if $\theta^{B}-\theta^{A}>\theta^{*}$ disclosures are positive and zero otherwise. And when $\theta^{B}-\theta^{A}$ decreases disclosures also diminish.

3. Infringement and Penalties: Our model has abstracted completely from infringement considerations. In fact, our set-up can be interpreted as follows. If $B$ obtains the innovation he can either obtain a patent or not. In the case he obtains a patent the model presumes that $B$ is automatically not infringing $A$ 's innovation. In other words, the model presumes that if $B$ 's innovation has a large enough inventive step to be patented will automatically escape infringement. However, in reality many innovations are patentable and also infringing (see, Scotchmer [16]), which means that so-called blocking patents may occur. The way to solve the problem is by means of licensing.

In the case that $B$ does not obtain a patent, the model presumes that his innovation does not infringe $A$ 's innovation. In other words, the model assumes that $B$ 's innovation has not a large enough inventive step to be patented but it will also be not infringing. We also recognize that we do not cover the case in which $B$ 's innovation is infringing and unpatentable. In this situation, if $B$ were to use his innovation in the market competition game he would have to pay infringement damages to the innovator.

In both cases, we conjecture that if the probability of the rival being found infringing $A$ 's innovation depends on the amount of knowledge disclosed, the new equilibrium configuration would be characterized by higher disclosures. Whether R\&D will be higher or not, it is an interesting question that we do not find easy to answer without an analytical model incorporating these additional features. These type of questions are left for future research.

\section{Concluding Remarks}

In this paper we have studied the relationships between disclosure, R\&D, IP and market interactions when disclosure is aimed at creating prior art. Here, we conclude with a brief discussion of our main 
results and potential extensions of our model.

Our results can be used from a policy perspective. We find that decreasing the patentability standards is not always a good policy choice. If the disclosure level is kept fixed, a lower patentability standard always increases the intensity of R\&D. However, this policy results in strategic withholding of knowledge on the part of first inventors when the knowledge externality has an important magnitude. Thus, a decrease in the patentability standards may finally hinder R\&D. In general, our results suggest that in those knowledge based industries, like Software, Computers and Semiconductors, 'strict' patentability standards may serve better the social goals of promoting disclosure of original innovations and leading to higher incremental invention.

On the market interactions side, we observe that when the knowledge externality is relatively low, a higher market premium leads to higher levels of disclosures and R\&D intensity. This result contradicts the common view that those industrial sectors with a high market premium should be characterized by a low level of disclosure and 'big' secrets. In this case a 'flourishing' environment characterized by 'highstakes-high-disclosure and high R\&D activity' arises. But we also underscore that when the knowledge externality is high enough the picture might look exactly the opposite: 'high-stakes-low-disclosure and low R\&D activity'. In this case, 'big' secrets may substantially hurt incremental innovation.

\section{Appendix}

ProOf OF LEMma 1. By part (a), we know that $f\left(d, \bar{s}, \pi^{B}\right):=f($.$) is a C^{1}$ function. Now, using our definition $\Delta_{V P}^{B}:=\pi^{H}-\pi^{M}$ and differentiating equation (1) with respect to $d$, we have:

$$
\gamma_{d}(d ; \bar{s}) \Delta_{V P}^{B}-C_{f f}(f(.), d) \frac{\partial f(.)}{\partial d}-C_{f d}(f(.), d)=0 \Rightarrow \frac{\partial f}{\partial d}(.)=\frac{\left[\gamma_{d}(d ; \bar{s}) \Delta_{V P}^{B}-C_{f d}(f(.), d)\right]}{C_{f f}(f(.), d)} \lessgtr 0
$$

Similarly differentiating equation (1) with respect to $\Delta_{V P}^{B}, \Delta_{V I}^{B}:=\pi^{M}-\pi^{L}$ and $\bar{s}$ we have:

$$
\begin{gathered}
\gamma(d ; \bar{s})-C_{f f}(f(.), d) \frac{\partial f(.)}{\partial \Delta_{V P}^{B}}=0 \Rightarrow \frac{\partial f}{\partial \Delta_{V P}^{B}}(.)=\left(C_{f f}(f(.), d)\right)^{-1} \gamma(d ; \bar{s})>0 \\
-C_{f f}(f(.), d) \frac{\partial f(.)}{\partial \Delta_{V I}^{B}}=0 \Rightarrow \frac{\partial f}{\partial \Delta_{V I}^{B}}(.)=\left(C_{f f}(f(.), d)\right)^{-1} \\
\frac{\partial \gamma(d ; \bar{s})}{\partial \bar{s}} \Delta_{V P}^{B}-C_{f f}(f(.), d) \frac{\partial f(.)}{\partial \bar{s}}=0 \Rightarrow \frac{\partial f}{\partial \bar{s}}(.)=\left(C_{f f}(f(.), d)\right)^{-1} \Delta_{V P}^{B} \frac{\partial \gamma(d ; \bar{s})}{\partial \bar{s}}>0
\end{gathered}
$$

ProOf OF PROPOSITION 1.(a) Notice that the constrained set $K$ is non-empty, closed and bounded. $K$ is non-empty because $\forall d \in \mathcal{D}$ there exists a unique $f$ that solves $B$ 's problem. $K$ is closed because $\gamma(d ; \bar{s})$ and $C_{f}(f, d)$ are continuous functions of $d$ and $f$. And it is bounded because both $f$ and $d \in[0,1]$ and hence $K \subset[0,1] \times[0,1]$. Therefore $K$ is a non-empty compact set. Given that 
$E U^{A}(f, d)=\left[(1-f) \pi^{H}+f W(d, \pi, \bar{s})\right]:[0,1] \times[0,1] \rightarrow \mathbb{R}$ is a continuous function of $d$ and $f$, by Weierstrass's theorem, $A$ 's problem has a solution.

(b) First by differentiating $\frac{\partial f\left(d, \bar{s}, \pi^{B}\right)}{\partial d}$ with respect to $d$ it is easily seen that a sufficient condition for $f\left(d, \bar{s}, \pi^{B}\right)$ to be a strictly convex function of disclosure is to suppose that:

Assumption A1: $\forall(f, d) \in[0,1] \times[0,1]: C_{f f d}(f, d)=0$ and $C_{f d d}(f, d) \leq 0$.

This last assumption says, that the rate at which the marginal cost increases is independent of $d$ and that the rate at which the marginal cost decreases with disclosures is itself a (weakly) decreasing function of $d$. The role played by Assumption 3 in characterizing B's best response is described in the following lemma.

Then, given Lemma 1 , the constrained set can be written as: $K:=\{(d, f) \in[0,1] \times[0,1]: f=$ $f(d ; \pi, \bar{s})\}$. By part (a) of the theorem there exists a pair $\left(d_{0}, f_{0}\right)$ such that:

$$
E U^{A}\left(\left(d_{0}, f_{0}\right)\right)=\operatorname{Max}\left\{E U^{A}(d, f):(d, f) \in K\right\}
$$

We define $v^{*}:=E U^{A}\left(\left(d_{0}, f_{0}\right)\right)$. Assume that there exists a $\left(d_{1}, f_{1}\right) \in K$ such that $d_{1} \neq d_{0}$ and $v^{*}=E U^{A}\left(\left(d_{1}, f_{1}\right)\right)$. Let $\theta \in(0,1)$ and define $\theta\left(d_{0}, f_{0}\right)+(1-\theta)\left(d_{1}, f_{1}\right)$. Then by quasiconcavity, $E U^{A}\left(\theta d_{0}+(1-\theta) d_{1}, \theta f_{0}+(1-\theta) f_{1}\right) \geq v^{*}$. However, observe that corresponding to $\theta d_{0}+(1-\theta) d_{1}$, there is a unique $f$ given by $f=f\left(\theta d_{0}+(1-\theta) d_{1} ; \pi, \bar{s}\right)$ such that $\left(\theta d_{0}+(1-\theta) d_{1}, f\left(\theta d_{0}+(1-\theta) d_{1} ; \pi, \bar{s}\right)\right) \in$ $K$. Then $f\left(\theta d_{0}+(1-\theta) d_{1} ; \pi, \bar{s}\right)<\theta f_{0}+(1-\theta) f_{1}$ because by Assumption A1, $f(d ; \pi, \bar{s})$ is a strictly convex function of $d$. Finally, because $E U^{A}(f, d)$ is a monotonically decreasing function of $f$,

$$
\begin{aligned}
E U^{A}\left(\theta d_{0}+(1-\theta) d_{1}, f\left(\theta d_{0}+(1-\theta) d_{1} ; \pi, \bar{s}\right)\right) & > \\
E U^{A}\left(\theta d_{0}+(1-\theta) d_{1}, \theta f_{0}+(1-\theta) f_{1}\right) & \geq v^{*}
\end{aligned}
$$

Thus, by contradicting the initial assumption that the pair $\left(d_{0}, f_{0}\right)$ is an equilibrium, we have shown uniqueness.

\section{First and Second Order Conditions}

To prove the rest of the propositions, we solve $A$ 's problem. We know from Lemma 1 that the constrained set can be written as:

$$
K:=\{(d, f) \in[0,1] \times[0,1]: f=f(d ; \pi, \bar{s})\}
$$

hence the maximization problem of the innovator is:

$$
\begin{aligned}
\max _{d \in[0,1]} E U^{A}(f(d ; \pi, \bar{s}), d) & =\max _{d \in[0,1]}\left[(1-f(d ; \pi, \bar{s})) \pi^{H}+f(d ; \pi, \bar{s}) W(d, \pi, \bar{s})\right] \\
\text { where } & : \quad W(d, \pi, \bar{s}):=\gamma(d ; \bar{s}) \pi^{L}+(1-\gamma(d ; \bar{s})) \pi^{M}
\end{aligned}
$$


and the first order (necessary) condition is:

$$
-\frac{\partial f}{\partial d}\left(d^{*}, \pi, \bar{s}\right) \pi^{H}+\frac{\partial f}{\partial d}\left(d^{*}, \pi, \bar{s}\right) W\left(d^{*}, \pi, \bar{s}\right)+f\left(d^{*}, \pi, \bar{s}\right) \frac{\partial W\left(d^{*}, \pi, \bar{s}\right)}{\partial d}=0
$$

then because $\frac{\partial W(d, \pi, \bar{s})}{\partial d}=-\gamma_{d}(d ; \bar{s})\left(\pi^{M}-\pi^{L}\right)$, we have the following FOC:

$$
\begin{aligned}
-\gamma_{d}(d ; \bar{s}) f\left(d^{*}, \pi, \bar{s}\right)\left(\pi^{M}-\pi^{L}\right) & =\frac{\partial f}{\partial d}\left(d^{*}, \pi, \bar{s}\right) \pi^{H}-\frac{\partial f}{\partial d}\left(d^{*}, \pi, \bar{s}\right) W\left(d^{*}, \pi, \bar{s}\right) \Longleftrightarrow M B^{*}= \\
-\gamma_{d}\left(d^{*} ; \bar{s}\right) f\left(d^{*}, \pi, \bar{s}\right) \Delta_{P R}^{A} & =\frac{\partial f}{\partial d}\left(d^{*}, \pi, \bar{s}\right)\left[\Delta_{M P}^{A}+\gamma\left(d^{*} ; \bar{s}\right) \Delta_{P R}^{A}\right]=M C^{*}
\end{aligned}
$$

The second order (local) condition that must be satisfied at $d^{*}$ is:

$$
H:=\left(\frac{\partial M C^{*}}{\partial d}-\frac{\partial M B^{*}}{\partial d}\right)>0
$$

Proof OF Proposition 2. Part (a) Using the FOC to differentiate $M B^{*}$ and $M C^{*}$ with respect to $\bar{s}$, we obtain ${ }^{12}$

$$
\frac{\partial M B^{*}}{\partial \bar{s}}=-\gamma_{d}\left(d^{*} ; \bar{s}\right) \frac{\partial f\left(d^{*}, \pi, \bar{s}\right)}{\partial \bar{s}} \Delta_{P R}^{A} ; \frac{\partial M C^{*}}{\partial \bar{s}}=\frac{\partial f}{\partial d}\left(d^{*}, \pi, \bar{s}\right) \frac{\partial \gamma\left(d^{*} ; \bar{s}\right)}{\partial \bar{s}} \Delta_{P R}^{A}
$$

because $\frac{\partial}{\partial \bar{s}}\left(\frac{\partial f}{\partial d}\left(d^{*}, \pi, \bar{s}\right)\right)=0$. By the Implicit Function Theorem: $\frac{\partial d^{*}(\pi, \bar{s})}{\partial \bar{s}}=\frac{1}{H}\left(\frac{\partial M B^{*}}{\partial \bar{s}}-\frac{\partial M C^{*}}{\partial \bar{s}}\right)$. Hence:

$$
\frac{\partial d^{*}(\pi, \bar{s})}{\partial \bar{s}}=\frac{\Delta_{P R}^{A}}{H}\left(-\gamma_{d}\left(d^{*} ; \bar{s}\right) \frac{\partial f\left(d^{*}, \pi, \bar{s}\right)}{\partial \bar{s}}-\frac{\partial f}{\partial d}\left(d^{*}, \pi, \bar{s}\right) \frac{\partial \gamma\left(d^{*} ; \bar{s}\right)}{\partial \bar{s}}\right)
$$

Using the expressions for $\frac{\partial f^{*}}{\partial \bar{s}}$ and $\frac{\partial f^{*}}{\partial d}$ from Lemma 1 we obtain:

$$
\frac{\partial d^{*}(\pi, \bar{s})}{\partial \bar{s}}=\frac{C_{f f}^{-1} \Delta_{P R}^{A} \frac{\partial \gamma(d ; \bar{s})}{\partial \bar{s}}}{H}\left[\left(-2 \gamma_{d}\left(d^{*} ; \bar{s}\right) \Delta_{V P}^{B}+C_{f d}(f(.), d)\right)\right]
$$

Thus:

If $-C_{f d}(f(), d)>.-2 \gamma_{d}\left(d^{*} ; \bar{s}\right) \Delta_{V P}^{B} \Rightarrow\left\{-2 \gamma_{d}\left(d^{*} ; \bar{s}\right) \Delta_{V P}^{B}+C_{f d}(f(), d).\right\}<0$ and because $\frac{C_{f f}^{-1} \Delta_{P R}^{A} \frac{\partial \gamma(d ; \bar{s})}{\partial \bar{s}}}{H}>0$, we have that $\frac{\partial d^{*}}{\partial \bar{s}}<0$.

Only if: $\frac{\partial d^{*}}{\partial \bar{s}}<0 \Rightarrow\left\{-2 \gamma_{d}\left(d^{*} ; \bar{s}\right) \Delta_{V P}^{B}+C_{f d}(f(), d).\right\}<0 \Rightarrow-C_{f d}(f(), d)>.-2 \gamma_{d}\left(d^{*} ; \bar{s}\right) \Delta_{V P}^{B}$.

Concerning the R\&D probability note: $f^{*}:=f\left(d^{*}(\pi, \bar{s}), \bar{s}, \pi\right)$. By the chain rule:

$$
\frac{\partial f^{*}}{\partial \bar{s}}=\frac{\partial f}{\partial d}\left(d^{*}(\pi, \bar{s}), \bar{s}, \pi\right) \frac{\partial d^{*}(\pi, \bar{s})}{\partial \bar{s}}+\frac{\partial f\left(d^{*}(\pi, \bar{s}), \bar{s}, \pi\right)}{\partial \bar{s}}
$$

\footnotetext{
${ }^{12}$ We assume, just to simplify, that $\gamma_{d \bar{s}}\left(d^{*} ; \bar{s}\right)=0$. All of our results hold no matter the sign of $\gamma_{d \bar{s}}\left(d^{*} ; \bar{s}\right)$.
} 
From Lemma 1: $\frac{\partial f^{*}}{\partial \bar{s}}>0$. Thus:

If: $-C_{f d}(f(), d)>.-2 \gamma_{d}\left(d^{*} ; \bar{s}\right) \Delta_{V P}^{B} \Rightarrow \frac{\partial d^{*}(\pi, \bar{s})}{\partial \bar{s}}<0$, and because $\frac{\partial f^{*}}{\partial d}>0 \Rightarrow \frac{\partial f^{*}}{\partial d} \frac{\partial d^{*}}{\partial \bar{s}}<0 \Longrightarrow$ $\frac{\partial f^{*}}{\partial \bar{s}} \lessgtr 0$.

Only if: $\frac{\partial f^{*}}{\partial \bar{s}} \lessgtr 0 \Rightarrow \frac{\partial f^{*}}{\partial d} \frac{\partial d^{*}}{\partial \bar{s}}<0$, and because $\frac{\partial f^{*}}{\partial d}>0 \Rightarrow \frac{\partial d^{*}}{\partial \bar{s}}<0 \Rightarrow-C_{f d}(f(), d)>$. $-2 \gamma_{d}(d ; \bar{s}) \Delta_{V P}^{B}$

ProOf OF PROPOSITION 3. Recalling that $\Delta_{M P}^{A}=\pi^{H}-\pi^{M}=\Delta_{V P}^{B}=\Delta_{M P}$ and using the FOC to differentiate $M B^{*}$ and $M C^{*}$ with respect to $\Delta_{M P}$, we obtain:

$$
\begin{aligned}
& \frac{\partial M B^{*}}{\partial \Delta_{M P}}=-\frac{\partial f\left(d^{*}, \pi, \bar{s}\right)}{\partial \Delta_{V P}^{B}} \gamma_{d}\left(d^{*} ; \bar{s}\right) \Delta_{P R}^{A} \\
& \frac{\partial M C^{*}}{\partial \Delta_{M P}}=\frac{\partial}{\partial \Delta_{V P}^{B}}\left(\frac{\partial f}{\partial d}\left(d^{*}, \pi, \bar{s}\right)\right)\left[\Delta_{M P}^{A}+\gamma\left(d^{*} ; \bar{s}\right) \Delta_{P R}^{A}\right]+\frac{\partial f}{\partial d}\left(d^{*}, \pi, \bar{s}\right)
\end{aligned}
$$

Then using the expression for $\frac{\partial f^{*}}{\partial d}$ from Lemma 1, it is direct to show that:

$$
\frac{\partial}{\partial \Delta_{V P}^{B}}\left(\frac{\partial f}{\partial d}\left(d^{*}, \pi, \bar{s}\right)\right)=\frac{\gamma_{d}\left(d^{*} ; \bar{s}\right)}{C_{f f}(f(.), d)}<0
$$

because by Assumption A.1: $\forall(f, d) \in[0,1] \times[0,1]: C_{f f d}(f(), d)=$.0 . Therefore using the Implicit Function Theorem:

$$
\frac{\partial d^{*}(\pi, \bar{s})}{\partial \Delta_{M P}}=\frac{1}{H}\left(-\gamma_{d}\left(d^{*} ; \bar{s}\right) \Delta_{P R}^{A} \frac{\partial f\left(d^{*}, \pi, \bar{s}\right)}{\partial \Delta_{V P}^{B}}-\frac{\gamma_{d}\left(d^{*} ; \bar{s}\right)}{C_{f f}(f(.), d)} \Pi^{A}\left(d, \bar{s}, \pi^{A}\right)-\frac{\partial f}{\partial d}\left(d^{*}, \pi, \bar{s}\right)\right)
$$

where $\Pi(d, \bar{s}, \pi)=\Delta_{M P}^{A}+\gamma\left(d^{*} ; \bar{s}\right) \Delta_{P R}^{A}$. Using the expressions of $\frac{\partial f^{*}}{\partial \Delta_{V P}^{B}}$ and $\frac{\partial f^{*}}{\partial d}$ from Lemma 1 in this last expression, we obtain:

$$
\frac{\partial d^{*}(\pi, \bar{s})}{\partial \Delta_{M P}}=\left\{\frac{-\gamma_{d}\left(d^{*} ; \bar{s}\right) \Delta_{P R}^{A} \gamma\left(d^{*} ; \bar{s}\right)-\gamma_{d}\left(d^{*} ; \bar{s}\right) \Pi\left(d^{*}, \bar{s}, \pi\right)-\left[\gamma_{d}\left(d^{*} ; \bar{s}\right) \Delta_{V P}^{B}-C_{f d}\left(f^{*}(.), d^{*}\right)\right]}{C_{f f} H}\right\}
$$

Part (a) If. For clarity reasons, we prove it in steps.

Step 1:If $-C_{f d}>-2 \gamma_{d} \Pi(.) \Rightarrow 0>C_{f d}-\gamma_{d}\left(\Delta_{M P}^{A}+\gamma(d ; \bar{s}) \Delta_{P R}^{A}\right)-\gamma_{d}(d ; \bar{s}) \Pi($.$) , because \Pi()=$. $\Delta_{M P}^{A}+\gamma\left(d^{*} ; \bar{s}\right) \Delta_{P R}^{A}$. Thus:

Step 2: $0>C_{f d}-\gamma_{d}\left(\Delta_{M P}^{A}+\gamma \Delta_{P R}^{A}\right)-\gamma_{d} \Pi()=.-\left[\gamma_{d} \Delta_{M P}^{A}-C_{f d}\right]-\gamma_{d} \gamma \Delta_{P R}^{A}-\gamma_{d} \Pi($.

$\Rightarrow \frac{\partial d^{*}(\pi, \bar{s})}{\partial \Delta_{M P}^{A}}<0$.

\section{Only if:}

Step 1: $\frac{\partial d^{*}}{\partial \Delta_{M P}^{A}}<0 \Rightarrow C_{f d}-\gamma_{d}\left(\Delta_{M P}^{A}+\gamma\left(d^{*} ; \bar{s}\right) \Delta_{P R}^{A}+\Pi().\right)<0 \Rightarrow$

Step 2:- $C_{f d}\left(f^{*}, d^{*}\right)>-\gamma_{d}\left(\Delta_{M P}^{A}+\gamma \Delta_{P R}^{A}+\Pi().\right)$. But $\Pi()=.\Delta_{M P}^{A}+\gamma\left(d^{*} ; \bar{s}\right) \Delta_{P R}^{A}$. Thus:

Step 3: $\frac{\partial d^{*}}{\partial \Delta_{M P}^{A}}<0 \Rightarrow-C_{f d}>-2 \gamma_{d} \Pi($.$) .$ 
With respect to the R\&D: $f^{*}:=f\left(d^{*}(\pi, \bar{s}), \bar{s}, \pi\right)$. By the chain rule:

$$
\frac{\partial f^{*}}{\partial \Delta_{M P}}=\frac{\partial f}{\partial d}\left(d^{*}(\pi, \bar{s}), \bar{s}, \pi\right) \frac{\partial d^{*}(\pi, \bar{s})}{\partial \Delta_{M P}}+\frac{\partial f\left(d^{*}(\pi, \bar{s}), \bar{s}, \pi\right)}{\partial \Delta_{M P}}
$$

From Lemma 1: $\frac{\partial f^{*}}{\partial \Delta_{M P}}=\frac{\partial f^{*}}{\partial \Delta_{V P}^{B}}>0$. Thus:

Step 1:(If) $-C_{f d}>-2 \gamma_{d} \Pi($.$) by part a) \frac{\partial d^{*}}{\partial \Delta_{M P}}<0$ and because $\frac{\partial f^{*}}{\partial d}>0 \Rightarrow \frac{\partial f^{*}}{\partial d} \frac{\partial d^{*}(\pi, \bar{s})}{\partial \Delta_{M P}}<0 \Longrightarrow$ $\frac{\partial f^{*}}{\partial \Delta_{M P}} \lessgtr 0$.

Only if: $\frac{\partial f^{*}}{\partial \Delta_{M P}} \lessgtr 0 \Rightarrow \frac{\partial f^{*}}{\partial d} \frac{\partial d^{*}(\pi, \bar{s})}{\partial \Delta_{M P}}<0$ and because $\frac{\partial f^{*}}{\partial d}>0 \Rightarrow \frac{\partial d^{*}}{\partial \Delta_{M P}}<0 \Rightarrow-C_{f d}()>$. $-2 \gamma_{d}(.) \Pi($.$) .$

Part (b). Again $f^{*}:=f\left(d^{*}(\pi, \bar{s}), \bar{s}, \pi\right)$. By the chain rule:

$$
\frac{\partial f^{*}}{\partial \Delta_{M P}}=\frac{\partial f}{\partial d}\left(d^{*}(\pi, \bar{s}), \bar{s}, \pi\right) \frac{\partial d^{*}(\pi, \bar{s})}{\partial \Delta_{M P}}+\frac{\partial f\left(d^{*}(\pi, \bar{s}), \bar{s}, \pi\right)}{\partial \Delta_{M P}}
$$

From Lemma 1: $\frac{\partial f^{*}}{\partial \Delta_{M P}}=\frac{\partial f^{*}}{\partial \Delta_{V P}}>0$. Thus If: $-C_{f d}>-2 \gamma_{d} \Pi(.) \Rightarrow \frac{\partial d^{*}}{\partial \Delta_{M P}} \geq 0$ and because $\frac{\partial f^{*}}{\partial d}>0 \Rightarrow \frac{\partial f^{*}}{\partial d} \frac{\partial d^{*}}{\partial \Delta_{M P}} \geq 0$. Thus, $\frac{\partial f^{*}}{\partial \Delta_{M P}}>0$.

ProOf OF PROPOSITION 4. Recalling that $\Delta_{P R}^{A}=\pi^{M}-\pi^{L}=\Delta_{V I}^{B}=\Delta_{P R}$ and using the FOC to differentiate $M B^{*}$ and $M C^{*}$ with respect to $\Delta_{P R}$, we obtain:

$$
\begin{aligned}
& \frac{\partial M B^{*}}{\partial \Delta_{P R}}=-\gamma_{d}\left(d^{*} ; \bar{s}\right) f\left(d^{*}, \pi, \bar{s}\right)-\gamma_{d}\left(d^{*} ; \bar{s}\right) \frac{\partial f\left(d^{*}, \pi, \bar{s}\right)}{\partial \Delta_{P R}^{A}} \Delta_{P R}^{A} ; \\
& \frac{\partial M^{*}}{\partial \Delta_{P R}}=\frac{\partial}{\partial \Delta_{V I}^{B}}\left(\frac{\partial f}{\partial d}\left(d^{*}, \pi, \bar{s}\right)\right) \Pi\left(d^{*}, \bar{s}, \pi\right)+\frac{\partial f}{\partial d}\left(d^{*}, \pi, \bar{s}\right) \gamma\left(d^{*} ; \bar{s}\right)
\end{aligned}
$$

Using the expression for $\frac{\partial f^{*}}{\partial d}$ that follows from Lemma 1 we have that:

$$
\frac{\partial}{\partial \Delta_{V I}^{B}}\left(\frac{\partial f}{\partial d}\left(d^{*}, \pi, \bar{s}\right)\right)=0
$$

Hence using the Implicit Function Theorem:

$$
\frac{\partial d^{*}(\pi, \bar{s})}{\partial \Delta_{P R}}=\frac{1}{H}\left[-\gamma_{d}\left(d^{*} ; \bar{s}\right) f\left(d^{*}, \pi, \bar{s}\right)-\gamma_{d}\left(d^{*} ; \bar{s}\right) \frac{\partial f\left(d^{*}, \pi, \bar{s}\right)}{\partial \Delta_{P R}^{A}} \Delta_{P R}^{A}-\frac{\partial f}{\partial d}\left(d^{*}, \pi, \bar{s}\right) \gamma\left(d^{*} ; \bar{s}\right)\right]
$$

From the FOC it follows that:

$$
-\gamma_{d}\left(d^{*} ; \bar{s}\right) f\left(d^{*}, \pi, \bar{s}\right)=\frac{\partial f}{\partial d}\left(d^{*}, \pi, \bar{s}\right)\left[\frac{\Delta_{M P}^{A}}{\Delta_{P R}^{A}}+\gamma\left(d^{*} ; \bar{s}\right)\right]
$$

Therefore:

$$
\frac{\partial d^{*}(\pi, \bar{s})}{\partial \Delta_{P R}}=\frac{1}{H}\left[\frac{\partial f}{\partial d}\left(d^{*}, \pi, \bar{s}\right) \frac{\Delta_{M P}^{A}}{\Delta_{P R}^{A}}-\gamma_{d}\left(d^{*} ; \bar{s}\right) \frac{\partial f\left(d^{*}, \pi, \bar{s}\right)}{\partial \Delta_{P R}^{A}} \Delta_{P R}^{A}\right]>0
$$


because by Lemma $1 \frac{\partial f^{*}}{\partial \Delta_{P R}}=\frac{\partial f^{*}}{\partial \Delta_{V I}^{B}}>0$.

With respect to the $\mathrm{R} \& \mathrm{D}$ probability: $f^{*}:=f\left(d^{*}(\pi, \bar{s}), \bar{s}, \pi\right)$. By the chain rule:

$$
\frac{\partial f^{*}}{\partial \Delta_{P R}}=\frac{\partial f}{\partial d}\left(d^{*}(\pi, \bar{s}), \bar{s}, \pi\right) \frac{\partial d^{*}(\pi, \bar{s})}{\partial \Delta_{P R}}+\frac{\partial f\left(d^{*}(\pi, \bar{s}), \bar{s}, \pi\right)}{\partial \Delta_{P R}}
$$

From Lemma 1: $\frac{\partial f^{*}}{\partial \Delta_{P R}}=\frac{\partial f^{*}}{\partial \Delta_{V I}^{B}}>0$ and because $\frac{\partial f^{*}}{\partial d}>0 \Rightarrow \frac{\partial f^{*}}{\partial d} \frac{\partial d^{*}}{\partial \Delta_{P R}}>0 \Longrightarrow \frac{\partial f^{*}}{\partial \Delta_{P R}}>0$.

\section{References}

[1] Allison, J. and M. Lemley (1998), "Empirical Evidence on the Validity of Litigated Patents," 26 AIPLA

[2] Anton, J. and D. Yao (2003), "Patents, Invalidity and the Strategic Transmission of Enabling Information," Journal of Economics \& Management Strategy, 12:2, pp. 151-178

[3] Anton, J. and D. Yao (2004), "Little Patents and Big Secrets: Managing Intellectual Property," Rand Journal of Economics, 35:1, pp. 1-22

[4] Baker, S., Lichtman D. and C. Mezzetti (2005), "Disclosure and Investment As Strategies in The Patent Race," The Journal of Law and Economics, vol. 48, pp. 173-194

[5] Bar, T. (2006), "Defensive Publications in an R\&D Race," Journal of Economics \& Management Strategy, Vol. 15, No. 1, pp. 229-254.

[6] Cohen W., Nelson R. and J. Walsh (2000), "Protecting Their Intellectual Assets: Appropriability Conditions and Why Manufacturing Firms Patent (or Not)," NBER WP 7552.

[7] Denicolo V. and L. Franzoni (2004), "Patents, Secrets and The First Inventor Defense," Journal of Economics \& Management Strategy, 13:3, pp. 517-538.

[8] Green, J. and S. Scotchmer (1995), "On the Division of Profit in Sequential Innovation," Rand Journal of Economics 26:1, pp. 20-33.

[9] Johnson, J. (2004), "Defensive Publishing by a Leading Firm," Johnson Graduate School of Management, Cornell University WP.

[10] Lanjouw, J. and M. Schankerman (2001), "Characteristics of Patent Litigation: a Window on Competition," Rand Journal of Economics 32:1, pp. 129-151.

[11] Lerner, J. (2000), "The Choice of Intellectual Property Protection: Evidence from Civil Litigation," Harvard University WP.

[12] Lichtman, D., Baker S. and K. Kraus (2000), "Strategic Disclosure in The Patent System," 53 Vanderbilt Law Review 2175. 
[13] Parchomovsky, G. (2000), "Publish or Perish," 18 Michigan Law Review.

[14] Ponce (2007), "More Secrecy...More Knowledge Disclosure? On Disclosure Outside of Patents," University Carlos III de Madrid WP.

[15] Scotchmer, S. and J. Green (1990), "Novelty and Disclosure in Patent Law," Rand Journal of Economics 21:1, pp. 131-146.

[16] Scotchmer, S.(2004), "Innovation and Incentives," The MIT Press. 